\title{
Multistage Interference Cancellation with Multipath Decorrelating for QPSK Asynchronous DS/CDMA System over Multipath Fading
}

\author{
Jianfeng Weng, Guoqiang Xue, Tho Le-Ngoc, and Sofiène Tahar \\ Dept. of Electrical \& Computer Engineering, Concordia University, Canada \\ email: $\{$ jfweng, xue, tho, tahar $\} @$ ece.concordia.ca
}

\begin{abstract}
A combined multistage interference cancellation (MIC) and multipath decorrelating scheme (MIC-DECO) for asynchronous QPSK DS/CDMA over frequency-selective multipath Rayleigh fading channels is introduced. Unlike the conventional MIC, which attempts to cancel the multiple-access interference (MAI) and total self interference (SI), the introduced scheme aims to remove the MAI, and partial SI incurred by the self intersymbol interference (SII). After cancellation, decorrelating is used first to separate the multipath signals and then to re-combine the resulting fading replicas for symbol decision. If the noise correlation in the fading replicas is known, an optimum combining structure (MIC-OPTM) can be achieved. Furthermore, when the MAI and SII are successfully removed, the MIC-OPTM can be replaced by the MIC using Rake combining (MIC-RAKE) with reduced complexity. The simulation results show that the MIC-DECO, MICOPTM, and MIC-RAKE in a multi-user environment provide a good performance close to the ideal performance in a single-user system, and outperform the conventional MIC even in the presence of channel estimation error.
\end{abstract}

\section{INTRODUCTION}

Multiuser decorrelating detection has been proposed to suppress the multiple-access interference (MAI) and the self interference (SI) for Direct-Sequence Code-Division Multiple Access (DS/ CDMA) systems in multipath fading channels $[1,2]$. Nevertheless, these detectors still require a large amount of computation, especially in asynchronous system when the numbers of active users and fading paths become large. On the other hand, various efforts on designing multistage interference cancellation (MIC) over multipath fading channels have been conducted in [3,4]. For instance, in [3], the MIC scheme was proposed to cancel the MAI, and a RAKE receiver was simulated to estimate the channel parameters and to combine the fading replicas for symbol decision in BPSK CDMA. However, apart from the MAI, the received signal was also corrupted by the SI caused by the multipath components of the desired user's signal. In order to remove the SI as well as the MAI, [4] considered the MIC scheme with the full SI cancellation. Nevertheless, note that the SI is made up of the self intersymbol interference (SII) incurred by the multipath components of the previous symbol and the self current symbol interference (SCI) which corresponds to the current symbol. Since the SCI contains the current symbol information which can be treated as useful signal for symbol decision, the MIC can proceed with the partial SI cancellation to cancel the SII alone rather than the full SI.

In this paper, we will introduce a MIC with multipath decorrelating (MIC-DECO) for QPSK asynchronous CDMA system over frequency-selective, Rayleigh multipath fading channels. Unlike the conventional MIC (MIC-CONV) [4], the introduced MIC considers the cancellation of the MAI and the partial SI. After cancellation, several combining techniques are considered. Note that for any user, the canceller output contains the superimposed multipath signals corresponding to the current symbol. Thus, the decorrelating operation can be used to separate those superimposed multipath signals before re-combining them. Such scheme is referred to as the MIC-DECO. Since the decorrelating operation in the MIC-DECO is conducted over multiple fading paths for each user, the computational complexity is reduced in comparison with the combined multiuser and multipath decorrelator in [2]. The MIC-DECO can also be applied to the system with time-varying spreading codes while the asynchronous decorrelator in [2] cannot. Furthermore, while in the MIC-CONV, the signal from other fading paths and bearing information of the current symbol was treated as interference to be removed, the MIC-DECO separates that multipath signal and uses it in the symbol decision. In this sense, the resulting performance would be better. The decorrelating operation may incur the correlation among the noise components in multiple fading paths. If the noise correlation is known to the receiver, a maximal ratio combining can be achieved. Although in practice, such an MIC scheme with optimum combining (MIC-OPTM) cannot be implemented due to the unavailability of the knowledge on the noise correlation, it can provide a performance benchmark for the MIC with various combining techniques. Furthermore, for a successful cancellation of the MAI and SII, the MIC-OPTM can be approximated by the MIC with Rake combining (MIC-RAKE).

Simulation results show that the MIC schemes with partial SI cancellation provide a good performance close to that in an ideal single-user system and outperform the MIC$\mathrm{CONV}$ even in the presence of the channel estimation error. Among the MIC schemes, the MIC-DECO can provide a slightly better performance than the MIC-RAKE in the initial stage, while in the $i$-th stage $(i>0)$, when the MAI and SII are successfully removed, the MIC-RAKE has a performance close to that of the MIC-OPTM and superior to that of the MIC-DECO. Furthermore, our analysis shows that the MIC-RAKE can inherently decorrelate the superimposed multipath fading replicas so that the decorrelating operation is not necessary. For this reason, the MIC-RAKE re- 
quires less computational complexity than the MIC-DECO.

\section{SySTEM DESCRIPTION}

Consider a QPSK asynchronous DS/CDMA system with $K$ active users over a frequency-selective multipath Rayleigh fading channel. The received signal, $r(t)$, can be written as

$$
r(t)=\sum_{k=1}^{K} \sqrt{\frac{2 \rho_{k}}{N}} \sum_{l=1}^{L_{k}} \alpha_{l}^{(k)} b_{k}\left(t-\tau_{l}^{(k)}\right) a_{k}\left(t-\tau_{l}^{(k)}\right)+z(t)
$$

where $\rho_{k}:=E_{k} / N_{o}$ is the normalized signal power, in which $E_{k}$ is the symbol energy for user $k$ and $N_{o}$ is the noise power spectral density. $L_{k}$ is the number of paths which can be either random or deterministic. $\alpha_{l}^{(k)}$ and $\tau_{l}^{(k)}$ are respectively the fading parameter and the time delay for the $l$-th path and user $k . z(t)$ is the background Gaussian noise with zero mean and unit power spectral density. $b_{k}(t)=\sum_{n=-\infty}^{\infty} e^{-j \phi_{n}^{(k)}} P_{T_{b}}\left(t-n T_{b}\right)$ is the information bearing waveform, where $\phi_{n}^{(k)}$ is the data bearing phase taken from the set $\{2 \pi(m-1) / 4, m=1,2, \ldots, 4\}$ and $P_{T}(t)$ is the rectangle waveform with duration $T$. $a_{k}(t)$ denotes the signature waveform given by

$$
a_{k}(t)=\sum_{i=-\infty}^{\infty} \frac{1}{\sqrt{2}}\left[a_{i}^{(k), I}+j a_{i}^{(k), Q}\right] P_{T_{c}}\left(t-i T_{c}\right)
$$

where $a_{i}^{(k), I}=a_{N+i}^{(k), I}$ and $a_{i}^{(k), Q}=a_{N+i}^{(k), Q}$ are respectively the in-phase and quadrature PN codes assigned to user $k$, which are taken from the set $\{-1,1\}$.

A channel estimator is used to estimate the channel parameters. The estimation model can be written for all $l$ and $k$ as

$$
\hat{\alpha}_{l}^{(k)}=\alpha_{l}^{(k)}+e_{l}^{(k)}
$$

where $e_{l}^{(k)}$ is the estimate noise in the $l$-th path for user $k$ and it is assumed statistically independent complex-valued Gaussian variable with zero mean and variance $\sigma_{e_{l k}}^{2}$ for all $l$ and $k$.

At the first part of the receiver, we assume that there are $\sum_{k=1}^{K} L_{k}$ correlators with the conjugate waveforms $\left\{a_{k}^{*}(t-\right.$ $\left.\left.\tau_{l}^{(k)}\right), l=1,2, \ldots, L_{k}\right\}$ to generate the decision variables for $K$ users. Consider the $q$-th fading path for user $u$, the received signal $r(t)$ is correlated with $\left\{a_{u}^{*}\left(t-\tau_{q}^{(u)}\right)\right\}$ and integrated from $n T_{b}+\tau_{q}^{(u)}$ to $(n+1) T_{b}+\tau_{q}^{(u)}$. Then, the output signal will be sampled at the time instant $(n+1) T_{b}+$ $\tau_{q}^{(u)}$. The resulting sample, denoted by $V_{n, q}^{(u)}$, is given by

$$
\begin{aligned}
V_{n, q}^{(u)}= & \sum_{k=1}^{K} \sqrt{2 \rho_{k}} \sum_{l=1}^{L_{k}} \alpha_{l}^{(k)}\left[e^{-j \phi_{n-m-1}^{(k)}} R_{k, u}\left(\tau_{k, l ; u, q}^{\prime}\right)\right. \\
& \left.+e^{-j \phi_{n-m}^{(k)}} \hat{R}_{k, u}\left(\tau_{k, l ; u, q}^{\prime}\right)\right]+\eta_{n, q}^{(u)}
\end{aligned}
$$

where $m:=\left\lfloor\left(\tau_{l}^{(k)}-\tau_{q}^{(u)}\right) / T_{b}\right\rfloor$, and $\tau_{k, l ; u, q}^{\prime}:=\left(\tau_{l}^{(k)}-\right.$ $\left.\tau_{q}^{(u)}\right)-m T_{b} . R_{k, u}(\tau)=\frac{1}{N} \int_{0}^{\tau} a_{k}(t-\tau) a_{u}^{*}(t) d t$ and $\hat{R}_{k, u}(\tau)=$ $\frac{1}{N} \int_{\tau}^{T_{b}} a_{k}(t-\tau) a_{u}^{*}(t) d t$ are the normalized partial correlation functions. $\eta_{n, q}^{(u)}$ is the noise component given by

$$
\eta_{n, q}^{(u)}=\frac{1}{\sqrt{N}} \int_{n T_{b}+\tau_{q}^{(u)}}^{(n+1) T_{b}+\tau_{q}^{(u)}} z(t) a_{u}^{*}\left(t-\tau_{q}^{(u)}\right) d t
$$

Since $z(t)$ is the Gaussian noise with zero mean and unit power spectral density, $\eta_{n, q}^{(u)}$ is Gaussian with zero mean and unit variance. The covariance of $\eta_{n_{1}, q_{1}}^{\left(u_{1}\right)}$ and $\eta_{n_{2}, q_{2}}^{\left(u_{2}\right)}$, i.e., $\frac{1}{2} E\left\{\eta_{n_{1}, q_{1}}^{\left(u_{1}\right)}\left[\eta_{n_{2}, q_{2}}^{\left(u_{2}\right)}\right]^{*}\right\}$, is set to $R_{u_{2}, u_{1}}\left(\tau_{2}^{L}-\tau_{1}^{S}\right)$ and $R_{u_{1}, u_{2}}^{*}\left(\tau_{1}^{L}-\tau_{2}^{S}\right)$, respectively, when $\tau_{1}^{S} \leq \tau_{2}^{L} \leq \tau_{1}^{L}$ and $\tau_{2}^{S} \leq \tau_{1}^{L} \leq \tau_{2}^{L}$. It is set to zero if $\tau_{2}^{S}>\tau_{1}^{L}$ or $\tau_{1}^{S}>\tau_{2}^{L}$, where $\tau_{i}^{S}=n_{i} T_{b}+\tau_{q_{i}}^{\left(u_{i}\right)}$ and $\tau_{i}^{L}=\tau_{i}^{S}+T_{b}$ for $i=1,2$.

For notational simplicity, $V_{n, q}^{(u)}$ of (4) will be written as

$V_{n, q}^{(u)}=\sqrt{2 \rho_{u}} \alpha_{q}^{(u)} e^{-j \phi_{n}^{(u)}}+S c_{n, q}^{(u)}+S i_{n, q}^{(u)}+M_{n, q}^{(u)}+\eta_{n, q}^{(u)}$

where $S c_{n, q}^{(u)}, S i_{n, q}^{(u)}$, and $M_{n, q}^{(u)}$ are defined as follows

$$
\begin{gathered}
S c_{n, q}^{(u)}=\sqrt{2 \rho_{u}} \sum_{l=1, l \neq q}^{L_{u}} \alpha_{l}^{(u)} e^{-j \phi_{n}^{(u)}} \ddot{R}_{u, u}\left(\tau_{u, l ; u, q}^{\prime}\right) \\
S i_{n, q}^{(u)}=\sqrt{2 \rho_{u}} \sum_{l=1, l \neq q}^{L_{u}} \alpha_{l}^{(u)}\left[d_{m+1} e^{-j \phi_{n-m-1}^{(u)}}\right. \\
\left.R_{u, u}\left(\tau_{u, l ; u, q}^{\prime}\right)+d_{m} e^{-j \phi_{n-m}^{(u)}} \hat{R}_{u, u}\left(\tau_{u, l ; u, q}^{\prime}\right)\right] \\
M_{n, q}^{(u)}=\sum_{k=1, k \neq u}^{K} \sqrt{2 \rho_{k}} \sum_{l=1}^{L_{k}} \alpha_{l}^{(k)}\left[e^{-j \phi_{n-m-1}^{(k)}}\right. \\
R_{k, u}\left(\tau_{k, l ; u, q}^{\prime}\right)+e^{\left.-j \phi_{n-m}^{(k)} \hat{R}_{k, u}\left(\tau_{k, l ; u, q}^{\prime}\right)\right]}
\end{gathered}
$$

In (8), $\ddot{R}_{u, u}(\tau)$ is set to either $R_{u, u}(\tau)$ or $\hat{R}_{u, u}(\tau)$ depending on $\tau_{l}^{(u)}<\tau_{q}^{(u)}$ or not. In (8), $d_{i}$ is an index function which can be set to 0 if $i=0$ and to 1 otherwise. $S c_{n, q}^{(u)}$ is referred to as the self current-symbol interference (SCI) and $S i_{n, q}^{(u)}$ the self intersymbol interference (SII). The sum of the SCI and the SII is the total SI. $M_{n, q}^{(u)}$ is the MAI.

From (6), we can see that the signal $e^{-j \phi_{n}^{(u)}}$ to be detected is submerged by the multiuser and multipath interferences. To suppress those interferences, various detectors can be designed as shown in the following.

\section{Multistage Interference CANCELlation}

In this section, we briefly review the conventional MIC with full SI cancellation [4]. Then, we discuss three MIC schemes using partial SI cancellation. 


\section{A. Conventional MIC with Full SI Cancellation}

The conventional MIC (MIC-CONV) with full SI cancellation [4] attempted to cancel the MAI and the total SI from the received signal in each stage of the MIC. The output of the canceller for the $q$-th branch in the $i$-th stage of the MIC is given by

$$
\begin{aligned}
V_{n, q}^{(u)}(i)= & V_{n, q}^{(u)}-\hat{S} c_{n, q}^{(u)}(i-1) \\
& -\hat{S} i_{n, q}^{(u)}(i-1)-\hat{M}_{n, q}^{(u)}(i-1)
\end{aligned}
$$

where $\hat{S} c_{n, q}^{(u)}(i-1), \hat{S} i_{n, q}^{(u)}(i-1)$, and $\hat{M}_{n, q}^{(u)}(i-1)$ are the reconstructed interference parts of $S c_{n, q}^{(u)}, S i_{n, q}^{(u)}$, and $M_{n, q}^{(u)}$, respectively. They can be calculated via (7), (8), and (9), respectively, by replacing $\alpha_{l}^{(k)}$ and $\phi_{n}^{(k)}$ by $\hat{\alpha}_{l}^{(k)}$ and $\hat{\phi}_{n}^{(k)}(i-1) . \hat{\alpha}_{l}^{(k)}$ is the channel estimate given by (3) and $\hat{\phi}_{n}^{(k)}(i-1)$ the tentative decision of $\phi_{n}^{(k)}$ in the $(i-1)$-th stage for the $n$-th symbol and user $k$.

As a result, if the subtraction can totally remove the SCI, the SII, and the MAI from $V_{n, q}^{(u)}(i)$ in (6), the resulting signal only contains the faded signal $\alpha_{q}^{(u)} \sqrt{2 \rho_{u}} e^{-j \phi_{n}^{(u)}}$ and the noise component $\eta_{n, q}^{(u)}$. Since there is no superimposed multipath signals, the Rake combining can be applied to collect the multiple fading replicas and the combining output in the MIC-CONV is given by

$$
X_{n}^{(u)}(i)=\left[\underline{\hat{\alpha}}^{(u)}\right]^{H} \underline{V}_{n}^{(u)}(i)
$$

where $\underline{V}_{n}^{(u)}(i)=\left[V_{n, 1}^{(u)}, V_{n, 2}^{(u)}, \ldots, V_{n, L_{u}}^{(u)}\right]^{T}$, and $\underline{\hat{\alpha}}^{(u)}=\left[\hat{\alpha}_{1}^{(u)}\right.$, $\left.\hat{\alpha}_{2}^{(u)}, \ldots, \hat{\alpha}_{L_{u}}^{(u)}\right]^{T}$, in which $\left\{\hat{\alpha}_{l}^{(u)}\right\}$ denote the channel estimates given by (3).

After computing the following decision variables,

$$
D_{p}(i)=\operatorname{Re}\left\{X_{n}^{(u)}(i) e^{j \psi_{p}}\right\}, \quad p=1,2, \ldots, 4
$$

where $\psi_{p}:=2 \pi(p-1) / 4$, the symbol is decided in favor of

$$
\hat{\phi}_{n}^{(u)}(i)=\psi_{p}=\operatorname{argmax}\left\{D_{p}(i)\right\}
$$

In the initial stage $(i=0)$, we let $V_{n, q}^{(u)}(0)=V_{n, q}^{(u)}$ and based on $V_{n, q}^{(u)}(0)$, the tentative decision $\hat{\phi}_{n}^{(u)}(0)$ can be readily obtained by following (11) to (13).

In the MIC-CONV, the SCI is regarded as an interference to be subtracted in (10). However, the SCI actually contains the current symbol, which can be shown useful for symbol decision. Hence, we may subtract partial SI instead of full SI. In the following, several MIC schemes with partial SI cancellation are discussed.

\section{B. MIC with Partial SI Cancellation}

\section{B.1 MIC with decorrelating (MIC-DECO)}

In the MIC-DECO, we proceed the MIC with the subtraction of the MAI and the partial SI (the SII part). The output signal in the $i$-th stage canceller is given by

$$
V_{n, q}^{(u)}(i)=V_{n, q}^{(u)}-\hat{S} i_{n, q}^{(u)}(i-1)-\hat{M}_{n, q}^{(u)}(i-1)
$$

where as in the MIC-CONV, $\hat{S} i_{n, q}^{(u)}(i-1)$ and $\hat{M}_{n, q}^{(u)}(i-1)$ are the reconstructed interference parts of $S i_{n, q}^{(u)}$ and $M_{n, q}^{(u)}$, respectively.

After subtraction, the resulting signal in (14) can be written using the vector notation as follows,

$$
\begin{aligned}
\underline{V}_{n}^{(u)}(i)= & \mathbf{R}_{s} \underline{\alpha}^{(u)} \sqrt{2 \rho_{u}} e^{-j \phi_{n}^{(u)}}+\underline{S}_{n}^{(u)}(i-1) \\
& +\underline{\tilde{M}}_{n}^{(u)}(i-1)+\underline{\eta}_{n}^{(u)}
\end{aligned}
$$

where

$$
\begin{array}{r}
\underline{\alpha}^{(u)}=\left[\alpha_{1}^{(u)}, \alpha_{2}^{(u)}, \ldots, \alpha_{L_{u}}^{(u)}\right]^{T} \\
\underline{\eta}_{n}^{(u)}=\left[\eta_{n, 1}^{(u)}, \eta_{n, 2}^{(u)}, \ldots, \eta_{n, L_{u}}^{(u)}\right]^{T} \\
\underline{\tilde{S}}_{n}^{(u)}(i-1)=\left[\tilde{S} i_{n, 1}^{(u)}(i-1), \ldots, \tilde{S} i_{n, L_{u}}^{(u)}(i-1)\right]^{T}, \\
\tilde{M}_{n}^{(u)}(i-1)=\left[\tilde{M}_{n, 1}^{(u)}(i-1), \ldots, \tilde{M}_{n, L_{u}}^{(u)}(i-1)\right]^{T}
\end{array}
$$

where $\tilde{S} i_{n, q}^{(u)}(i-1)=S i_{n, q}^{(u)}-\hat{S} i_{n, q}^{(u)}(i-1)$ and $\tilde{M}_{n, q}^{(u)}(i-1)=$ $M_{n, q}^{(u)}-\hat{M}_{n, q}^{(u)}(i-1)$.

In (15), $\mathbf{R}_{s}$ is an $L_{u} \times L_{u}$ correlation matrix of the signature waveforms. Its $i j$-th entry is set to $\hat{R}_{u, u}\left(\tau_{j}^{(u)}-\tau_{i}^{(u)}\right)$ if $\tau_{j}^{(u)} \geq \tau_{i}^{(u)}$ and $R_{u, u}\left(T_{b}+\tau_{j}^{(u)}-\tau_{i}^{(u)}\right)$ otherwise. By (5), the correlation matrix of $\underline{\eta}_{n}^{(u)}$ can be recognized to be $\mathbf{R}_{s}$.

It is well known that the decorrelating operation is very useful in separating the multiuser and multipath signals [2]. By pre-multiplying (15) with $\mathbf{R}_{s}$ inverse, i.e., $\mathbf{R}_{s}^{-1}$, the multipath signals can be separated. We thus have

$$
\mathbf{U}(i)=\mathbf{R}_{s}^{-1} \underline{V}_{n}^{(u)}(i)=\underline{\alpha}^{(u)} \sqrt{2 \rho_{u}} e^{-j \phi_{n}^{(u)}}+\underline{w}_{n}^{(u)}(i)
$$

where $\underline{w}_{n}^{(u)}(i)=\mathbf{R}{ }_{s}^{-1}\left[\underline{\tilde{S}}_{n}^{(u)}(i-1)+\underline{\tilde{M}}_{n}^{(u)}(i-1)+\underline{\eta}_{n}^{(u)}\right]$.

After decorrelating the multipath signals, there are various methods to combine the resulting fading replicas in $\mathbf{U}(i)$. Here, we may directly combine those multipath fading replicas as in [1]. The combining output is given by

$$
X_{n}^{(u)}(i)=\left[\underline{\hat{\alpha}}^{(u)}\right]^{H} \mathbf{U}(i)
$$

Further symbol decision can proceed by following (12)(13).

The above decorrelating operation in the MIC-DECO is conducted over multiple fading paths for each user rather than for all users and multipath. Thus, the whole computational complexity is somehow reduced in comparison to the combined multiuser and multipath asynchronous decorrelator in [2]. On the other hand, compared with the MICCONV [4], where the SCI was treated as an interference to 
be subtracted, the MIC-DECO separates that multipath signal and uses it in symbol decision. In this way, the resulting performance would be better.

The combining method in (18) is not optimum due to the fact that the noise vector $\underline{w}_{n}^{(u)}(i)$ is correlated. If we know the correlation a priori, the optimum combining can be used to provide the maximal ratio combining after the noise whitening [2] as shown in the following.

\section{B.2 MIC with optimum combining (MIC-OPTM)}

Let $\mathbf{R}_{w}^{(u)}(i)$ denote the correlation matrix of $\underline{w}_{n}^{(u)}(i)$. The optimum combining output can be given by

$$
X_{n}^{(u)}(i)=\left[\underline{\hat{\alpha}}^{(u)}\right]^{H}\left[\mathbf{R}_{w}^{(u)}(i)\right]^{-1} \mathbf{U}(i)
$$

The symbol decision in the $i$-th stage may follow (12) and (13). Note that here the term "optimum" does not mean the optimum detection. Rather, it refers to the optimum combining after the MIC. Accordingly, the detection method is named the MIC-OPTM.

Although theoretically the MIC-OPTM is possible to conduct, it cannot be implemented in practice inasmuch as the correlation matrix of $\underline{w}_{n}^{(u)}(i)$ is unknown to the receiver or difficult to be estimated. Nevertheless, the MIC-OPTM may provide a performance benchmark for the MIC with all other combining techniques. Furthermore, it can be shown that when the interference (i.e., the SII and the MAI) are successfully removed, the MIC-OPTM can be approximated by the MIC with Rake combining (MIC-RAKE).

\section{B.3 MIC with Rake combining (MIC-RAKE)}

Assuming that for $i>0$, the multipath and multiuser interferences are successfully subtracted (setting $\underline{S}_{n}^{(u)}(i-$ $1)+\underline{\tilde{M}}_{n}^{(u)}(i-1)$ to zero in $\underline{w}_{n}^{(u)}(i)$ of $\left.(17)\right)$, we note that the correlation matrix of $\underline{w}_{n}^{(u)}(i)$ reduces to $\left[\mathbf{R}_{s}\right]^{-1}$. Thus, the output signal, after the noise whitening and the maximal ratio combining, is given by

$$
X_{n}^{(u)}(i)=\left[\underline{\hat{\alpha}}^{(u)}\right]^{H}\left[\mathbf{R}_{s}\right] \mathbf{U}(i)=\left[\underline{\hat{\alpha}}^{(u)}\right]^{H} \underline{V}_{n, q}^{(u)}(i)
$$

Following (12) and (13), we may obtain the symbol decision in the $i$-th stage.

The final step in (20) is actually the Rake combining (MIC-RAKE). Therefore, the result in (20) suggests that when the SII and the MAI are totally removed, the combining output of the MIC-OPTM is equivalent to that obtained by the MIC-RAKE. In other words, as long as the SII and MAI are effectively canceled, the MIC-RAKE can provide performance better than the MIC-DECO. Further derivations of the performance bounds of the MIC-RAKE scheme can be found in [5].

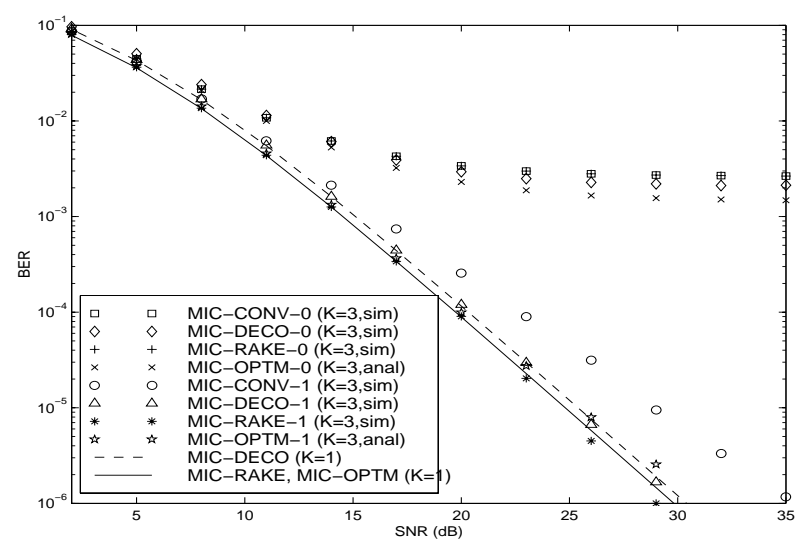

Fig. 1. Performance comparison of the MIC schemes in a 3-user system over a 2-path Rayleigh fading channel. ( $N=31$, equal-power users. Perfect channel knowledge).

\section{Illustrative Results}

Without loss of generality, we assume that the first user is the user of interest.

System model: Gold sequences with $N=31$ are considered as the PN codes. We consider identical fading channels with $\mathrm{E}\left\{\left[\alpha_{l}^{(k)}\right]^{2}\right\}=2$ for all fading paths. Total SNR\#k per bit is $\frac{1}{2} L_{k} 2 \rho_{k} \mathrm{E}\left\{\left[\alpha_{l}^{(k)}\right]^{2}\right\} / \log _{2} M=\rho_{k} L_{k} . L_{k}=L$ is assumed the same for all users. The multipath fading channel is considered to be a tapped delay line with $T_{c} / 2$ delay [6]. The time delay for user $k$ in the $l$-th path is $\tau_{l}^{(k)}=T^{(k)}+l T_{c} / 2$, where $T^{(k)}$ is set to $(k-1) T_{b} / 10 \bmod T_{b} .3 \times 10^{6}$ Monte Carlo trials are conducted to estimate the BER.

Results: For notational simplicity, let MIC-NAME- $i$ denote the performance of the MIC-NAME which relies on the $i$-th stage decision. In the initial stage (MIC-NAME-0), no interference cancellation is used. Evidently, the MICRAKE-0 is identical to the conventional RAKE receiver [6].

In the following, we first consider the performance of the MIC with perfect channel knowledge.

Fig. 1 compares the simulation results on the BER of the MIC schemes in a 3-user system with $\mathrm{SNR}=20 \mathrm{~dB}$ over a 2-path frequency selective identical Rayleigh fading channel. Equal-power users are considered. As a benchmark, the analytical single-user BERs for the MIC-DECO and the MIC-RAKE are provided in the figure. Also, the analytical results for the MIC-OPTM are plotted for comparison. It can be seen that the 1-stage MIC (RAKE, DECO, and $\mathrm{CON}$ ) can provide a significant performance improvement over the 0 -stage counterparts, in which no interference cancellation scheme is used. In the 1-stage MIC schemes, both the MIC-RAKE-1 and the MIC-DECO-1 can provide an improvement in SNR of about $2.5 \mathrm{~dB}$ at a BER of $10^{-4}$, as compared to the MIC-CONV-1. Besides, we also note that the MIC-RAKE has the same single-user performance as the MIC-OPTM and outperforms the MIC-DECO. In the 


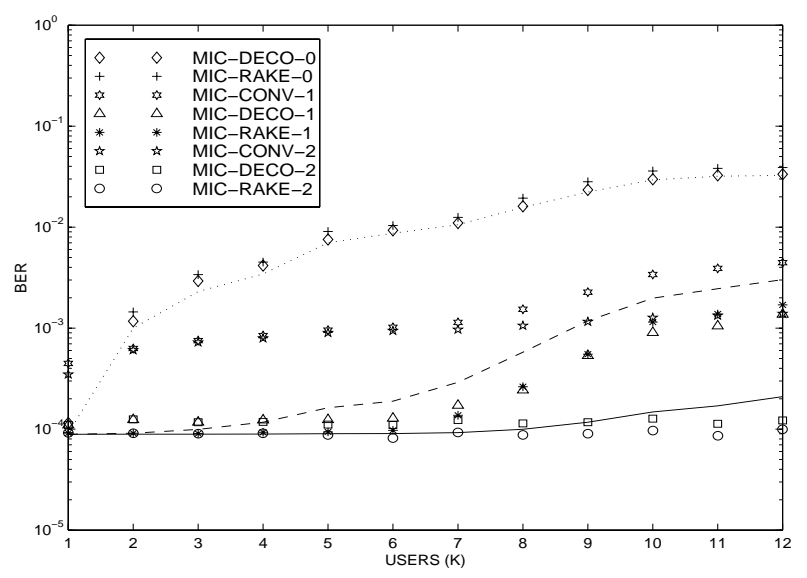

Fig. 2. Simulation results on the BER of the MIC-CONV, the MIC-DECO, and the MIC-RAKE in a multi-user system over a 2-path Rayleigh fading channel. SNR=20dB, $N=31$, and equal-power users. Perfect channel knowledge. Dotted line: MIC-OPTM-0, dashed line: MICOPTM-1, solid line: MIC-OPTM-2.

multiuser system (K=3), by effectively subtracting the interferences, the canceller output is similar to that in the singleuser system and thus the MIC-RAKE-1 still conducts better than the MIC-DECO-1 as shown in the figure. However, the performance difference is very small (approximately $0.5 \mathrm{~dB}$ at the level of $\mathrm{BER}=10^{-4}$ ). When there is no interference cancellation (at the stage 0 ), the MIC-OPTM-0 cannot be approximated by the MIC-RAKE- 0 . Both the MIC-RAKE0 and the MIC-DECO-0 are not optimum and the MICDECO-0 has a slightly better performance than the MICRAKE-0 at high SNR (SNR $>15 \mathrm{~dB}$ ). Generally, they can provide a performance comparable to that obtained by the MIC-OPTM-0. Finally, we note that the performance of the MIC-OPTM-1 seems worse than those of the MIC-RAKE1 and the MIC-DECO-1. This phenomenon might be due to the Gaussian approximation in modeling the residual MAI and SI to obtain the analytical BER for the MIC-OPTM-1.

In Fig. 2, we plot the simulation results on the BER of the MIC-CONV, the MIC-DECO, and the MIC-OPTM in a multi-user system with SNR=20dB over a 2-path Rayleigh fading channel versus the number of users. The MIC-CONV0 has the same performance to that of the MIC-RAKE- 0 and therefore is not plotted in the figure. The analytical results for the MIC-OPTM-0,1,2 are also plotted. The results show that in the presence of multiple users, the MIC-DECO-1,2 still outperform the detector without interference cancellation (MIC-DECO-0) and provide a performance close to that of the MIC-OPTM-1,2. Besides, it can be seen that the simulation results are well lower bounded by the corresponding analytical results of the MIC-OPTM for the initial stage $(i=0)$. As $i$ increases to 1 or 2 , deviation may occur especially for large $K$. A possible explanation for this is that when $K$ becomes large, the MAI also increases and thus the correlation among the bit error rates for users

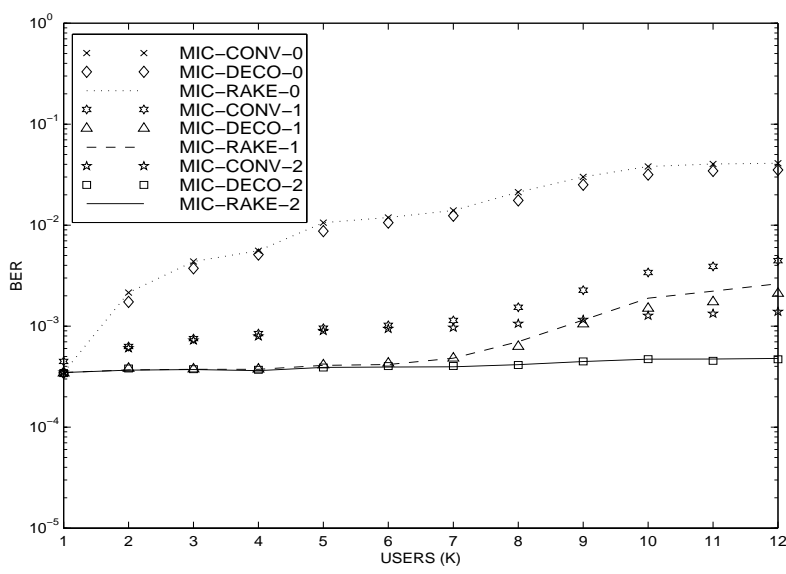

Fig. 3. Simulation results on the BER of the MIC-CONV, the MIC-DECO and the MIC-RAKE in a multi-user system over a 2-path Rayleigh fading channel. $(\mathrm{SNR}=20 \mathrm{~dB}, N=31$, equal-power users. Imperfect channel knowledge, $\left.\sigma_{e}^{2}=0.01\right)$.

in the previous stage may be salient. Thus, when we ignore such correlation and use the Gaussian approximation to model the residual interferences, performance difference occurs especially for large $K$. Note that in the 0-th stage, the Gaussian approximation may work well and the MICOPTM-0 may provide a good lower bound for the results of the other MIC schemes.

Next, the channel estimation error is considered and the variance of the channel estimation in (3) is assumed same for all $l$ and $u$ and denoted by $\sigma_{e}^{2}$.

Comparison of the simulation results of the MIC-CONV, the MIC-DECO and the MIC-RAKE is shown in Fig. 3, where $\sigma_{e}^{2}=0.01$. We can see that in the presence of channel estimation error, the MIC-DECO and MIC-RAKE have a similar performance and outperform the MIC-CONV.

\section{ACKNOWLEDGMENT}

This work is partially supported by Ericsson Research Canada.

\section{REFERENCES}

[1] D. Chen and S. Roy, "An adaptive multiuser receiver for CDMA systems," IEEE J. Select. Areas Commun., vol. SAC-12, pp. 808-816, June 1994.

[2] Z. Zvonar, "Combined multiuser detection and diversity reception for wireless CDMA systems," IEEE Trans. Vehi. Tech., vol. VT-45, pp. 205-211, Feb. 1996.

[3] S. Striglis, A. Kaul, N. Yang, and B. D. Woerner, "A multistage RAKE receiver for improved capacity of CDMA systems," in IEEE Vehi. Tech. Conf., VTC94, pp. 789-793, 1994.

[4] Y. C. Yoon, R. Kohno, and H. Imai, "Cascaded co-channel interference cancelling and diversity combining for spread spectrum multiaccess over multipath fading channels," IEICE Trans. Commun., vol. E76-B, pp. 163-168, Feb. 1993.

[5] J. F. Weng, G. Q. Xue, T. Le-Ngoc, and S. Tahar, "Multistage interference cancellation with diversity reception for QPSK asynchronous DS/CDMA system over multipath fading," in ICC99, Vancouver, Canada, June 6-10 1999.

[6] J. G. Proakis, Digital communications. New York: McGraw-Hill, 1995. 